\title{
Bivariate generalized Pareto distribution for extreme atmospheric particulate matter
}

\begin{abstract}
The high particulate matter (PM10) level is the prominent issue causing various impacts to human health and seriously affecting the economics. The asymptotic theory of extreme value is apply for analyzing the relation of extreme PM10 data from two nearby air quality monitoring stations. The series of daily maxima PM10 for Johor Bahru and Pasir Gudang stations are consider for year 2001 to 2010 databases. The $85 \%$ and $95 \%$ marginal quantile apply to determine the threshold values and hence construct the series of exceedances over the chosen threshold. The logistic, asymmetric logistic, negative logistic and asymmetric negative logistic models areconsidered as the dependence function to the joint distribution of a bivariate observation. Maximum likelihood estimation is employed for parameter estimations. The best fitted model is chosen based on the Akaike Information Criterion and the quantile plots. It is found that the asymmetric logistic model gives the best fitted model for bivariate extreme PM10 data and shows the weak dependence between two stations.
\end{abstract}

Keyword: Bivariate; Generalized Pareto distribution; Goodness of fit; PM10 